\title{
Hard probe radiative energy loss beyond soft-gluon approximation
}

\author{
Bojana Blagojevic $^{* a}$, Magdalena Djordjevic $^{a}$ and Marko Djordjevic ${ }^{b}$ \\ ${ }^{a}$ Institute of Physics Belgrade, University of Belgrade \\ Belgrade 11 000, Serbia \\ ${ }^{b}$ Faculty of Biology, Institute of Physiology and Biochemistry, University of Belgrade \\ Belgrade 11 000, Serbia \\ E-mail: bojanabeipb.ac.rs, magda@ipb.ac.rs, dmarko@bio.bg.ac.rs
}

One of the widely used kinematic assumptions in calculating hard probe radiative energy loss within QGP, is the soft-gluon approximation, which considers that energy loss of the parent parton via gluon's bremsstrahlung is small compared to its initial energy. However, diverse theoretical formalisams obtained a notable energy loss of high $p_{\perp}$ partons, implicitly suggesting inadequacy of this approximation.

To address this issue, we relax the soft-gluon approximation within the DGLV formalism. Although the obtained analytic expressions are quite more involved compared to the soft-gluon case, the numerical predictions are surprisingly nearly indistinguishable in these two cases. Additionally, we also obtained that the above conclusion is robust with respect to variations of the presumed longitudinal distance distribution of scattering centers. Consequently, the results presented here provide confidence that, regardless of the concerns mentioned above, surprisingly, the soft-gluon approximation can reliably be applied within the DGLV formalism. Finally, we discuss generalizing the relaxation in the dynamical medium as well, which ensures broader relevance of the conclusions obtained here.

International Conference on Hard and Electromagnetic Probes of High-Energy Nuclear Collisions 30 September - 5 October 2018

Aix-Les-Bains, Savoie, France

${ }^{*}$ Speaker. 


\section{Introduction}

One of the most common assumptions in calculating radiative energy loss of high $p_{\perp}$ partons is the soft-gluon $(\mathrm{sg})$ approximation, which assumes that the radiated gluon energy $(\omega)$ is much smaller $\left(x=\frac{\omega}{E} \ll 1\right)$ than the initial parton energy $(E)$. This approximation was widely used in various theoretical models $[1,2,3,4]$, which obtained considerable radiative energy loss, suggesting the inadequacy of the approximation. On that note, some of these models went beyond the softgluon (bsg) approximation [5, 6, 7], but reported inconsistent conclusions on the adequacy of this assumption. Contrarily, our dynamical energy loss model [8], by applying the same approximation, obtained very good agreement with the extensive set of experimental suppression data [8,9], implicitly indicating the adequacy of such approximation. Nevertheless even within this dynamical model, the approximation is not applicable in the intermediate momentum range $\left(5<p_{\perp}<10\right.$ $\mathrm{GeV}$ ), and primarily for gluon jets, as gluons lose notably more energy compared to quarks, due to the relative color factor of $9 / 4$. Therefore, the question of validity of the soft-gluon approximation ( $\mathrm{sga}$ ) remained still unresolved, which we systematically reconsider in [10] for gluon-jet radiative energy loss within the DGLV [11] formalism to the 1st order in opacity. Its generalization to the case when a dynamical medium is considered is also discussed in that paper. Additionally, we address the robustness of our conclusions, by taking into account two opposite limits of scattering centers longitudinal distribution: the exponential and the uniform one. It is worth noting that, these proceedings contain only the main results, while for more details, we refer the reader to [10].

\section{Analytical and numerical results}

We relax the sga in radiative energy loss calculations within the DGLV [11] formalism, which considers: finite size and an optically thin QGP consisting of static scattering centers. The jetmedium interactions are modelled by a static color-screened Yukawa potential (Eq.(5) from [12]), while, according to [13], gluons in a finite $T$ QCD medium are considered to be transversely polarized with effective mass given by $m_{g}=\mu / \sqrt{2}$, where $\mu$ is Debye mass. On that note, in [10] we calculated 11 Feynman diagrams for gluon jets, by allowing $x$ to acquire a finite value and under the following assumptions: $i$ ) that consistently for all diagrams, the direction of flight of the initial gluon is the longitudinal one; ii) the soft-rescattering approximation and iii) the $1^{\text {st }}$ order in opacity approximation.

Under the common assumption of an exponentially distributed scattering centers $\frac{2}{L} e^{-2 \frac{z_{1}-z_{0}}{L}}$ (which mimics a rapidly evolving medium, e.g. in [11]), and after involving calculations (for details see [10]), we obtain the $b s g$ expression for the single gluon radiation spectrum:

$$
\begin{aligned}
& \quad \frac{d N_{g, b s g}^{(1), e x p}}{d x}=\frac{C_{2}(G) \alpha_{s}}{\pi} \frac{L}{\lambda} \frac{\left(1-x+x^{2}\right)^{2}}{x(1-x)} \int \frac{d^{2} \mathbf{q}_{1}}{\pi} \frac{\mu^{2}}{\left(\mathbf{q}_{1}^{2}+\mu^{2}\right)^{2}} \int d \mathbf{k}^{2} \\
& \times\left\{\frac{\left(\mathbf{k}-\mathbf{q}_{1}\right)^{2}+\chi}{\left(\frac{4 x(1-x) E}{L}\right)^{2}+\left(\left(\mathbf{k}-\mathbf{q}_{1}\right)^{2}+\chi\right)^{2}}\left(2 \frac{\left(\mathbf{k}-\mathbf{q}_{1}\right)^{2}}{\left(\mathbf{k}-\mathbf{q}_{1}\right)^{2}+\chi}-\frac{\mathbf{k} \cdot\left(\mathbf{k}-\mathbf{q}_{1}\right)}{\mathbf{k}^{2}+\chi}-\frac{\left(\mathbf{k}-\mathbf{q}_{1}\right) \cdot\left(\mathbf{k}-x \mathbf{q}_{1}\right)}{\left(\mathbf{k}-x \mathbf{q}_{1}\right)^{2}+\chi}\right)\right. \\
& \left.+\frac{\mathbf{k}^{2}+\chi}{\left(\frac{4 x(1-x) E}{L}\right)^{2}+\left(\mathbf{k}^{2}+\chi\right)^{2}}\left(\frac{\mathbf{k}^{2}}{\mathbf{k}^{2}+\chi}-\frac{\mathbf{k} \cdot\left(\mathbf{k}-x \mathbf{q}_{1}\right)}{\left(\mathbf{k}-x \mathbf{q}_{1}\right)^{2}+\chi}\right)+\left(\frac{\left(\mathbf{k}-x \mathbf{q}_{1}\right)^{2}}{\left(\left(\mathbf{k}-x \mathbf{q}_{1}\right)^{2}+\chi\right)^{2}}-\frac{\mathbf{k}^{2}}{\left(\mathbf{k}^{2}+\chi\right)^{2}}\right)\right\}
\end{aligned}
$$




$$
=\frac{C_{2}(G) \alpha_{s}}{\pi} \frac{L}{\lambda} \int \frac{d^{2} \mathbf{q}_{1}}{\pi} \frac{\mu^{2}}{\left(\mathbf{q}_{1}^{2}+\mu^{2}\right)^{2}} \int d \mathbf{k}^{2} f_{b s g}^{\text {exp }}\left(\mathbf{k}, \mathbf{q}_{1}, x\right),
$$

where $\mathbf{k}$ and $\mathbf{q}_{1}$ are the transverse momentum of radiated and exchanged gluons, respectively; $\alpha_{s}$ is the strong coupling constant, $\lambda$ the mean free path, $L$ the medium length and $\chi=m_{g}^{2}\left(1-x+x^{2}\right)$. To the extent of our knowledge, this result presents the first introduction of the effective gluon mass in the bsga radiative energy loss. Note that, only a part of $\frac{d N_{g, b s g}^{(1)}}{d x}$, corresponding to the jet-radiated gluon interaction, i.e. $f\left(\mathbf{k}, \mathbf{q}_{1}, x\right)$, is altered by this relaxation, and in the $s g$ had the form (from [11] for gluon jets):

$$
f_{s g}^{\exp }\left(\mathbf{k}, \mathbf{q}_{1}, x\right)=\frac{1}{x} \frac{\left(\mathbf{k}-\mathbf{q}_{1}\right)^{2}+m_{g}^{2}}{\left(\frac{4 x E}{L}\right)^{2}+\left(\left(\mathbf{k}-\mathbf{q}_{1}\right)^{2}+m_{g}^{2}\right)^{2}} 2\left\{\frac{\left(\mathbf{k}-\mathbf{q}_{1}\right)^{2}}{\left(\mathbf{k}-\mathbf{q}_{1}\right)^{2}+m_{g}^{2}}-\frac{\mathbf{k} \cdot\left(\mathbf{k}-\mathbf{q}_{1}\right)}{\mathbf{k}^{2}+m_{g}^{2}}\right\} .
$$

It is straightforward to show that Eq. (2.1) is symmetric under the exchange of radiated $(k)$ and final $(p)$ gluons, as anticipated, due to the inability to distinguish between two identical gluons, and that for an infinitesimally small $x$ recovers the $s g$ limit given by Eq. (2.2).

Next we address how the $s g a$ relaxation affects our $R_{A A}$ [14] predictions (for predictions for additional observables see [10]). The numerical procedure for generating the bare gluon quenched spectrum is thoroughly explained in [10], where the following standard parameters values are used: $\alpha_{s}=\frac{g_{s}^{2}}{4 \pi}=0.3, L=5 \mathrm{fm}, \lambda=1 \mathrm{fm}, \mu=\sqrt{4 \pi \alpha_{s}\left(1+n_{f} / 6\right)} T, n_{f}=3$ and $T=300 \mathrm{MeV}$, to mimic the LHC conditions. From Fig. 1 we unexpectedly (based on the expressions analysis above) observe nearly overlapping $R_{A A}$ predictions for the $b s g$ and $s g$ cases.
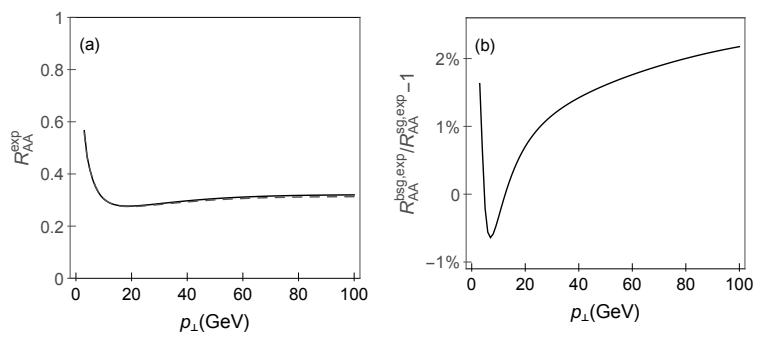

Figure 1: (a) Comparison of gluon-jet $R_{A A}$ in $b s g$ (solid line) with the one in $s g$ (dashed line) case as a function of $p_{\perp}$, when exponential distribution of scattering centers is taken into account. (b) The relative change of $R_{A A}$ with respect to the $s g$ limit. Adapted from [10].

\section{Sensitivity to the longitudinal distance distribution}

To examine the robustness of our results, next we test the sensitivity of the conclusions from Section 2 to the presumed longitudinal distance distribution between scattering centers. On that note, we apply the opposite limit to the one studied in the previous section, i.e. the uniform distribution, and in the $b s g$ case obtain (for details see [10]):

$$
f_{b s g}^{u n i}\left(\mathbf{k}, \mathbf{q}_{1}, x\right)=\frac{\left(1-x+x^{2}\right)^{2}}{x(1-x)}\left\{( 1 - \frac { \operatorname { s i n } ( \frac { ( \mathbf { k } - \mathbf { q } _ { 1 } ) ^ { 2 } + \chi } { 2 x ( 1 - x ) E } L ) } { \frac { ( \mathbf { k } - \mathbf { q } _ { 1 } ) ^ { 2 } + \chi } { 2 x ( 1 - x ) E } L } ) \frac { 1 } { ( \mathbf { k } - \mathbf { q } _ { 1 } ) ^ { 2 } + \chi } \left(2 \frac{\left(\mathbf{k}-\mathbf{q}_{1}\right)^{2}}{\left(\mathbf{k}-\mathbf{q}_{1}\right)^{2}+\chi}-\frac{\mathbf{k} \cdot\left(\mathbf{k}-\mathbf{q}_{1}\right)}{\mathbf{k}^{2}+\chi}\right.\right.
$$




$$
\begin{aligned}
& \left.-\frac{\left(\mathbf{k}-\mathbf{q}_{1}\right) \cdot\left(\mathbf{k}-x \mathbf{q}_{1}\right)}{\left(\mathbf{k}-x \mathbf{q}_{1}\right)^{2}+\chi}\right)+\left(1-\frac{\sin \left(\frac{\mathbf{k}^{2}+\chi}{2 x(1-x) E} L\right)}{\frac{\mathbf{k}^{2}+\chi}{2 x(1-x) E} L}\right) \frac{1}{\mathbf{k}^{2}+\chi}\left(\frac{\mathbf{k}^{2}}{\mathbf{k}^{2}+\chi}-\frac{\mathbf{k} \cdot\left(\mathbf{k}-x \mathbf{q}_{1}\right)}{\left(\mathbf{k}-x \mathbf{q}_{1}\right)^{2}+\chi}\right) \\
& \left.+\left(\frac{\left(\mathbf{k}-x \mathbf{q}_{1}\right)^{2}}{\left(\left(\mathbf{k}-x \mathbf{q}_{1}\right)^{2}+\chi\right)^{2}}-\frac{\mathbf{k}^{2}}{\left(\mathbf{k}^{2}+\chi\right)^{2}}\right)\right\},
\end{aligned}
$$

while in the $s g$ case it acquired the form (from [15] for gluon jets and a static medium):

$$
f_{s g}^{u n i}\left(\mathbf{k}, \mathbf{q}_{1}, x\right)=\frac{1}{x}\left\{\left(1-\frac{\sin \left(\frac{\left(\mathbf{k}-\mathbf{q}_{1}\right)^{2}+m_{g}^{2}}{2 x E} L\right)}{\frac{\left(\mathbf{k}-\mathbf{q}_{1}\right)^{2}+m_{g}^{2}}{2 x E} L}\right) \frac{1}{\left(\mathbf{k}-\mathbf{q}_{1}\right)^{2}+m_{g}^{2}} 2\left(\frac{\left(\mathbf{k}-\mathbf{q}_{1}\right)^{2}}{\left(\mathbf{k}-\mathbf{q}_{1}\right)^{2}+m_{g}^{2}}-\frac{\mathbf{k} \cdot\left(\mathbf{k}-\mathbf{q}_{1}\right)}{\mathbf{k}^{2}+m_{g}^{2}}\right\},\right.
$$

where the notation is the same as in the previous section. Again we observe that Eq. (3.1) is symmetric under the exchange of radiated $(k)$ and final $(p)$ gluons, and that for $x \ll 1$ it reduces to the $s g$ limit given by Eq. (3.2). Since the $\frac{d N_{g, b s g}^{(1)}}{d x}$ expression for a uniform distribution (obtained from Eq. (3.1)) is notably different than its exponential analogon (Eq. (2.1)), we assess also for this case the effect of the sga relaxation on the numerical predictions. Analogous to Section 2, we obtain Fig. 2 for the uniform distribution, and by comparing Figs. 1 and 2 we infer that numerical results for these two opposite longitudinal distance distribution limits are practically identical.
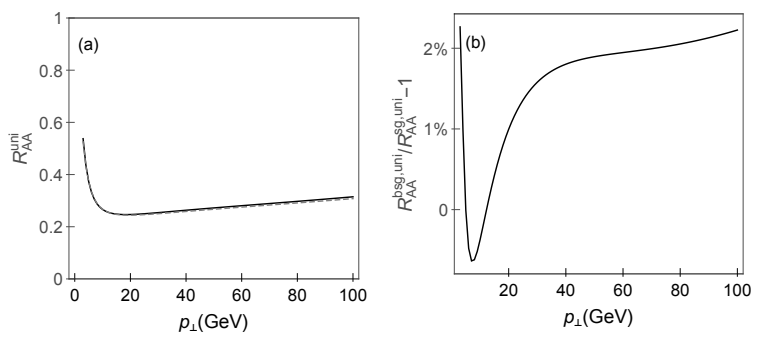

Figure 2: (a) Comparison of gluon-jet $R_{A A}$ in $b s g$ (solid line) with the one in $s g$ (dashed line) case as a function of $p_{\perp}$, when uniform distribution of scattering centers is taken into account. (b) The quantification of the effect and its expression in percentage. Adapted from [10].

\section{Conclusions}

In order to address the adequacy of the $s g a$, first we considered exponential distribution of scattering centers, and obtained that gluon's $b s g$ expression is apparently different, and considerably more complicated than in the $s g$ case. However, we surprisingly obtained that the numerical predictions in these two cases are nearly indistinguishable. Due to the relative color factor of 4/9, high $p_{\perp}$ quarks are less likely to be affected by this relaxation. This implies that, within the DGLV formalism, the sga remains valid. Furthermore, by applying the opposite, i.e. the uniform distribution, we obtained that the conclusions regarding the importance of the sga in the DGLV formalism are robust to the presumed longitudinal distance distribution. As an outlook, we also expect that the sga can be reliably applied to the dynamical energy loss formalism, which still remains to be explicitly tested in the future. 


\section{Acknowledgments}

This work is supported by the European Research Council, grant ERC-2016-COG: 725741, and by the Ministry of Science and Technological Development of the Republic of Serbia, under project numbers ON171004 and ON173052.

\section{References}

[1] N. Armesto, C.A. Salgado and U.A. Wiedemann, Medium induced gluon radiation off massive quarks fills the dead cone, Phys. Rev. D 69 (2004) 114003 [hep-ph/ 0312106 ].

[2] R. Baier, Y.L. Dokshitzer, A.H. Mueller, S. Peigne and D. Schiff, Radiative energy loss and $p(T)$ broadening of high-energy partons in nuclei, Nucl. Phys. B 484 (1997) 265 [hep-ph/9608322].

[3] M. Gyulassy, P. Levai and I. Vitev, Reaction operator approach to nonAbelian energy loss, Nucl. Phys. B 594 (2001) 371 [nucl-th/ 0006010 ].

[4] A. Majumder and M. Van Leeuwen, The Theory and Phenomenology of Perturbative QCD Based Jet Quenching, Prog. Part. Nucl. Phys. A 66 (2011) 41 [arXiv: 1002.2206$].$

[5] L. Apolinario, L., N. Armesto and C.A.Salgado, Medium-induced emissions of hard gluons, Phys. Lett. B 718 (2012) 160 [arXiv:1204.2929].

[6] B.W. Zhang and X.N. Wang, Multiple parton scattering in nuclei: Beyond helicity amplitude approximation, Nucl.Phys. A 720 (2003) 429 [hep-ph/0301195].

[7] G. Ovanesyan and I. Vitev, An effective theory for jet propagation in dense QCD matter: jet broadening and medium-induced bremsstrahlung, JHEP bf 1106 (2011) 080 [arXiv:1103.1074]; Medium-induced parton splitting kernels from Soft Collinear Effective Theory with Glauber gluons, Phys. Lett. B bf 706 (2012) 371 [arXiv:1109.5619].

[8] M. Djordjevic and M. Djordjevic, LHC jet suppression of light and heavy flavor observables, Phys. Lett. B 734 (2014) 286 [arXiv: 1307.4098 ].

[9] M. Djordjevic, M. Djordjevic and B. Blagojevic, RHIC and LHC jet suppression in non-central collisions, Phys. Lett. B 737 (2014) 298 [arXiv: 1405 .4250].

[10] B. Blagojevic, M. Djordjevic and M. Djordjevic, Calculating hard probe radiative energy loss beyond soft-gluon approximation: how valid is the approximation?, (Phys. Rev. C, in Press) (2018) [arXiv:1804.07593].

[11] M. Djordjevic and M. Gyulassy, Heavy quark radiative energy loss in QCD matter, Nucl. Phys. A 733 (2004) 265 [nucl-th/ 0310076$].$

[12] M. Gyulassy and X.N. Wang, Multiple collisions and induced gluon Bremsstrahlung in QCD, Nucl. Phys. B 420 (1994) 583 [nucl-th/9306003].

[13] M. Djordjevic and M. Gyulassy, The Ter-Mikayelian effect on QCD radiative energy loss, Phys. Rev. C 68 (2003) 034914 [nucl-th/ 0305062 ].

[14] A. Dainese, Perspectives for the study of charm in-medium quenching at the LHC with ALICE, Eur. Phys. J. C 33 (2004) 495 [nucl-ex/ 0312005 ].

[15] M. Djordjevic, Theoretical formalism of radiative jet energy loss in a finite size dynamical QCD medium, Phys. Rev. C 80 (2009) 064909 [arXiv: 0903.4591 ]. 